\title{
Review Article \\ Potential Role of Meiosis Proteins in Melanoma Chromosomal Instability
}

\author{
Scott F. Lindsey, ${ }^{1}$ Diana M. Byrnes, ${ }^{1}$ Mark S. Eller, ${ }^{1}$ Ashley M. Rosa, ${ }^{1}$ Nitika Dabas, ${ }^{1}$ \\ Julia Escandon, ${ }^{1}$ and James M. Grichnik ${ }^{1,2,3,4}$ \\ ${ }^{1}$ Department of Dermatology and Cutaneous Surgery, University of Miami Miller School of Medicine, Miami, FL 33136, USA \\ ${ }^{2}$ Anna Fund Melanoma Program Sylvester Comprehensive Cancer Center, University of Miami Miller School of Medicine, \\ Miami, FL 33136, USA \\ ${ }^{3}$ Frankel Family Division of Melanocytic Tumors, Department of Dermatology and Cutaneous Surgery, \\ University of Miami Miller School of Medicine, Miami, FL 33136, USA \\ ${ }^{4}$ Interdisciplinary Stem Cell Institute, University of Miami Miller School of Medicine, University of Miami, FL 33136, USA
}

Correspondence should be addressed to James M. Grichnik; grichnik@miami.edu

Received 29 March 2013; Accepted 21 May 2013

Academic Editor: Giuseppe Argenziano

Copyright (C) 2013 Scott F. Lindsey et al. This is an open access article distributed under the Creative Commons Attribution License, which permits unrestricted use, distribution, and reproduction in any medium, provided the original work is properly cited.

\begin{abstract}
Melanomas demonstrate chromosomal instability (CIN). In fact, CIN can be used to differentiate melanoma from benign nevi. The exact molecular mechanisms that drive CIN in melanoma have yet to be fully elucidated. Cancer/testis antigens are a unique group of germ cell proteins that are found to be primarily expressed in melanoma as compared to benign nevi. The abnormal expression of these germ cell proteins, normally expected only in the testis and ovaries, in somatic cells may lead to interference with normal cellular pathways. Germ cell proteins that may be particularly critical in CIN are meiosis proteins. Here, we review pathways unique to meiosis with a focus on how the aberrant expression of meiosis proteins in normal mitotic cells "meiomitosis" could impact chromosomal instability in melanoma and other cancers.
\end{abstract}

\section{Introduction}

Melanomas exhibit chromosomal instability (CIN). In fact, CIN is one of the most useful molecular markers to differentiate melanomas from benign nevi $[1,2]$. Bastian et al. found that $96.2 \%$ of melanomas demonstrated chromosomal aberrations while only $13.0 \%$ of benign nevi showed these same abnormalities, of which all were Spitz nevi with stable 11p duplications [1]. Melanomas generally exhibit an increased number of overall chromosomes with frequent large translocations [1].

The fact that melanomas have such unstable genomes comes as no surprise, as genomic instability is widely regarded as the hallmark of cancer [3-7]. While genomic instability decreases the viability of most cells, it may also permit a subpopulation of cells to acquire genetic changes that lead them to escape normal growth control mechanisms. In addition, genomic instability allows established cancers to evolve and evade immunologic and pharmacologic destruction [8]. The extent to which different mechanisms play a role in genomic instability is controversial [4]; however three pathways are generally most accepted. These are defective DNA repair, telomere crisis, and mitotic spindle malfunction [3, 9-12]. An underappreciated but potentially important research area is the abnormal expression of germ cell proteins.

Expression of germ cell proteins has long been observed in cancer cells [13]. Cancer/testis antigens (CTAs) are a family of germ cell proteins expressed in a multitude of different histological tumor types $[13,14]$. These proteins have been noted to have both diagnostic and prognostic value [2]. Studies have shown that expression of specific CTAs in melanoma can be used to predict tumor thickness, the presence of ulceration, and likelihood to undergo metastasis [15]. Melanomas have also been shown to express meiosis specific proteins including SCP1 (homologous chromosome pairing) [16], HORMAD1 (meiotic synapse regulation) [17], SPO11 (double 
stranded DNA breaks) [18], and REC8 (meiosis cohesion protein) [2]. We have also found three of these meiosis proteins, addition to a fourth not previously described, to be present in melanoma using western blot analysis (Figure 1) and immunofluorescence (Figure 2). Although the potential impact of the expression of meiotic proteins during mitosis in causing CIN has been suggested [17-21] and a role in reductional division in irradiated polyploid cells has been noted [22], thus far there has been no research directly evaluating the potential direct role of meiosis proteins in the creation of CIN. This review is focused on understanding meiosis pathways and explaining how the expression of these pathways during mitosis ("meiomitosis" $[19,20])$ may result in chromosomal instability.

\section{Meiosis General Overview: What Makes Meiosis Different from Mitosis?}

Meiosis is a specialized type of cell division. In contrast to mitosis in which a diploid cell $(2 \mathrm{~N})$ duplicates its DNA once and divides to produce two genetically identical diploid cells $(2 \mathrm{~N})$, in meiosis a diploid cell $(2 \mathrm{~N})$ duplicates its DNA once and undergoes two distinct rounds of cell division to produce four genetically unique haploid (1N) cells (Table 1). While mitosis occurs in all somatic cells of the human body, meiosis occurs naturally only in the male testis and female ovary.

The meiosis pathways are thought to have evolved from double-stranded DNA repair pathways [23]. In fact, meiosis still functions as a DNA repair process. This occurs through recombination events, where corresponding sections of DNA on the two homologous chromosomes are exchanged. This ensures that DNA damage is not passed on to progeny [24, $25]$, in addition to the obvious role of producing genetic diversity to facilitate species evolution [25-27].

Mitosis and meiosis have many similarities (Table 2). The second division of meiosis (meiosis II) has many aspects that are nearly identical to mitosis. The first division, on the other hand, exhibits three major modifications: (i) meiotic pairing to allow recombination, (ii) kinetochore coorientation to allow for homologous chromosome segregation during anaphase I, and (iii) stepwise loss of cohesion to ensure that sister chromatids segregate together in meiosis I [28]. These unique meiosis I pathways are the ones most likely to interfere with normal mitosis and will be the primary focus of this review.

\section{Meiosis Gene Activation: The Regulatory Switch}

The switch to meiosis from mitosis occurs in germ cells when the decision is made to produce gametes. Prior to this switch, germ cells use mitotic divisions as a way of selfrenewal to increase their numbers and ensure the cell line does not become depleted [29]. The decision for cells to transition from a mitotic to meiotic cell cycle is complex and differs vastly among organisms. In most simple single-celled organisms that undergo asexual reproduction, this transition occurs as a result of environmental stressors. In yeast, nutrient deprivation triggers the dephosphorylation of the
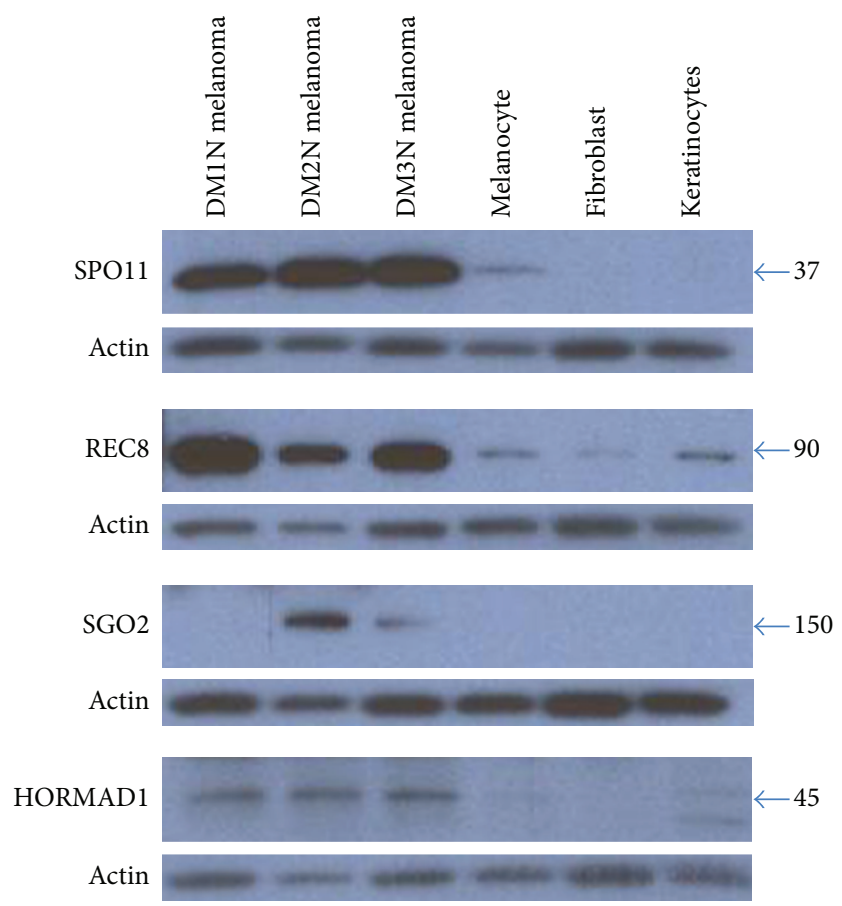

FIGURE 1: Analysis of meiosis proteins by western blot analysis. Western blot analysis was used to measure the expression of SPO11 (Abcam, ab81695), REC8 (Sigma, HPA031729), SGO2 (Sigma, HPA035163), and HORMAD1 (Abcam, ab57883) in melanoma lines compared to nontransformed melanocytes, fibroblasts, and keratinocytes. The cytoplasmic $\beta$-actin for each experiment is shown below its respective blot as a loading control.

RNA binding protein Mei2 by Pat1 Kinase which initiates the switch from mitosis to meiosis [30]. In Caenorhabditis elegans germline cells, GLD1 (quaking), GLD2 (Poly(A) polymerase), and GLD3 (Bicaudal C) have been shown to be critical for this switch $[31,32]$. In mammals, the decision to transition from mitosis to meiosis is yet more complex and there are numerous regulatory molecules that govern this change. STRA8 is one of these proteins and is known to be imperative for the switch from mitosis to meiosis in both human male and female germ lines [33]. Data from Li et al. support this notion, showing that the spermatocytes in mice with depleted STRA8 failed to enter meiosis [34].

The crucial point in the cell cycle by which the cell must decide to make the mitotic-to-meiotic shift is at the G1/S checkpoint [35]. This is because the cell must undergo meiotic-specific events during $S$ phase to ensure the proper progression of meiosis. The meiotic cohesin protein REC8 (discussed further below) must be incorporated into the newly replicated DNA during the premeiotic $S$ phase so that the kinetochores can orient appropriately to allow for reduction division during meiosis I.

Although meiosis follows mitosis in the production of gametes, it is important to note that in the fertilized egg, mitosis is reestablished after meiosis. Thus the mitosis-meiosis pathways can be switched on and off and are reversible. Dysregulation of this switch causing the collision of the pathways could certainly cause CIN. 

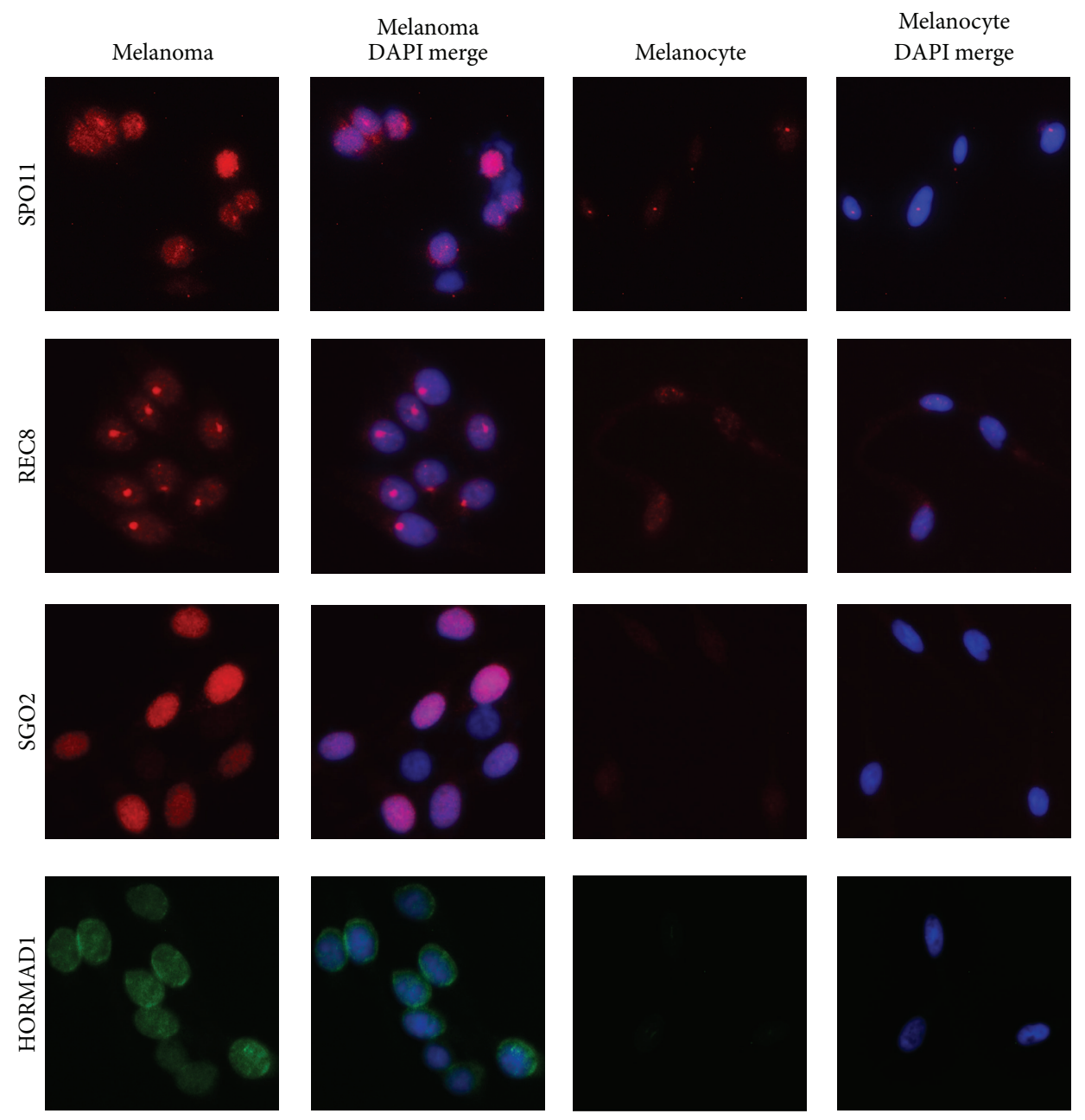

FIGURE 2: Analysis of meiosis proteins by immunofluorescence. Immunofluorescence was used to analyze the expression of SPO11, REC8, SGO2, and HORMAD1 in the DM2N melanoma line and nontransformed melanocytes using the same antibodies listed in Figure 1. Antirabbit secondary antibody was used for SPO11, REC8, and SGO2 and anti-mouse secondary was used for HORMAD1. The staining patterns varied between the different antibodies. SPO11, REC8, and SGO2 stained in a predominately nuclear manner with SPO11 demonstrating a fibular, dotted pattern, REC8 with discrete nuclear dots, and SGO2 with diffuse staining throughout the nucleus. HORMAD1 had a mostly cytoplasmic staining pattern although nuclear staining was also visualized. With all four antibodies, the staining in the melanoma was markedly stronger than the melanocytes.

\section{DNA Double Strand Breaks: Rotating the Genes}

The formation of crossover events lies at the heart of meiosis, as it allows for genetic recombination between homologous chromosomes and is necessary for the proper alignment of chromosomes during meiosis I. Crossovers are initiated by the creation of double-stranded breaks (DSBs) by the protein SPO11 [36].

SPO11 is a homolog of the archeal type II topoisomerase A subunit [37]. SPO11 creates DSBs using a catalytic tyrosine to attack the phosphodiester backbone of DNA, creating a covalent bond between itself and the $5^{\prime}$ end of the break [37]. Each SPO11 protein associates with only one strand of DNA; thus two SPO11 molecules are needed for each DSB [37]. Once the DSB is formed, SPO11 is removed by polymerase $\beta$ with the help of the MRE11 complex $[36,38]$ and the $5^{\prime}$ ends of the breaks are excised, leaving stretches of $3^{\prime}$ ssDNA [36]. This single stranded DNA associates with DMC1 and RAD51, two proteins known to be involved in doublestranded DNA repair, to form a filament [37]. This filament then begins the search for a location of homology on the opposite chromosome.

Many of the factors which ensure that the single stranded DNA correctly associates with a corresponding DNA fragment on the homologous chromosome have yet to be elucidated. It is clear that the meiosis specific kinase MEK1 plays a role in ensuring interhomolog binding through its interaction with RAD54 and RAD51 [39, 40]. MEK1 also promotes recombination by suppressing DSB repairs [41]. Upon identification of the appropriate homologue, DMC1 begins the crossover recombination event using one of the $3^{\prime}$ ends to mediate a stable invasion of the homologous chromosome [36]. DNA synthesis then extends the end of the 
TABLE 1: Summary of the major events in each stage of the meiotic and mitotic cell cycle.

\begin{tabular}{|c|c|c|c|}
\hline $\begin{array}{l}\text { Stage of meiotic } \\
\text { division }\end{array}$ & Outcome & $\begin{array}{l}\text { Stage of mitotic } \\
\text { division }\end{array}$ & Outcome \\
\hline S Phase I & $\begin{array}{l}\text { Duplication of genetic material, DNA double strand } \\
\text { breaks induced by SPO11 }\end{array}$ & S Phase & Duplication of genetic material \\
\hline Prophase I & $\begin{array}{l}\text { Chromosome condensation, homologous } \\
\text { recombination }\end{array}$ & Prophase & Chromosome condensation \\
\hline Metaphase I & $\begin{array}{l}\text { Tetrad alignment at metaphase plate, cohesin degraded } \\
\text { from chromosome arms but remains at centromere, } \\
\text { monooriented sister kinetochores }\end{array}$ & Metaphase & $\begin{array}{l}\text { Chromosome alignment at metaphase } \\
\text { plate, cohesin degraded from } \\
\text { centromere, bioriented sister } \\
\text { kinetochores }\end{array}$ \\
\hline Anaphase I & $\begin{array}{l}\text { Homologous chromosomes separate to opposite sides } \\
\text { of dividing cell, sister chromatids remain attached }\end{array}$ & Anaphase & $\begin{array}{l}\text { Sister chromatids separate to opposite } \\
\text { sides of dividing cell }\end{array}$ \\
\hline Telophase I & $\begin{array}{l}\text { Chromatid decondensation } \\
\text { (sometimes just partial decondensation) }\end{array}$ & Telophase & $\begin{array}{l}\text { Chromatid decondensation, two } \\
\text { daughter cells are diploid }\end{array}$ \\
\hline Prophase II & $\begin{array}{l}\text { No chromosome duplication, chromosome } \\
\text { recondensation }\end{array}$ & & \\
\hline Metaphase II & $\begin{array}{l}\text { Sister chromatids align at metaphase plate, cohesin } \\
\text { degraded from centromere, bi-oriented sister } \\
\text { kinetochores }\end{array}$ & & \\
\hline Anaphase II & $\begin{array}{l}\text { Sister chromatids separate to opposite sides of dividing } \\
\text { cell }\end{array}$ & & \\
\hline Telophase II & $\begin{array}{l}\text { Chromosome decondensation, four daughter cells are } \\
\text { haploid }\end{array}$ & & \\
\hline
\end{tabular}

TABLE 2: Summary of the differences between meiosis I, meiosis II, and mitosis.

\begin{tabular}{|c|c|c|c|}
\hline & Meiosis I & Meiosis II & Mitosis \\
\hline Reductional division & Yes & No & No \\
\hline Equational division & No & Yes & Yes \\
\hline Daughter Cells Genetically Identical & No & No & Yes \\
\hline Double strand breaks introduced by SPO11 & Yes & No & No \\
\hline Pol $\beta$ required for meiotic recombination and chromosome synapsis & Yes & No & No \\
\hline Homologous chromosome recombination and segregation & Yes & No & No \\
\hline Degradation of cohesion along chromosome arms & Yes & Not present & Not present \\
\hline Degradation of cohesion at centromeres & No & Yes_during anaphase II & Yes-during anaphase \\
\hline SGO2 protection of REC8 located at pericentromeric regions & Yes & No & No \\
\hline
\end{tabular}

invading strand. By recapture of this strand, a joint molecule is generated that contains a Holiday Junction, which can then be resolved into either a noncrossover or crossover event [36].

HORMAD1 also plays a critical role in synapsis formation through its role in DSB formation [42]. It ensures that adequate number DSBs are formed to allow for successful homology search [42]. Independently, HORMAD1 also promotes the proper formation of the synaptonemal complex, a proteinaceous structure which stabilizes the tetrad and ensures proper homolog pairing [42].

Together these factors cause DNA strand breaks and create a controlled number of crossover events. Deregulation, or simply the presence, of these pathways in otherwise mitotic cells would be expected to cause translocations as well as insertions and deletions. Although there is no data specifically implicating these pathways in cancer, it is important to note that SPO11 [18], DMC1 [22], and HORMAD1 [17] have all been shown to be increased in cancer. Both SPO11 and
HORMAD1 have been specifically noted in melanoma [17, 18] and we found them to be overexpressed in melanoma compared to nontransformed cell types using western blot analysis (Figure 1) and immunofluorescence (Figure 2).

\section{DNA Polymerase $\beta$ : Potential Role Evolving the Genome}

At least sixteen DNA polymerases exist in eukaryotes, each with its own unique role in maintaining the integrity of the genome [43]. Specific high fidelity DNA polymerases function in replicating the genome during the $S$ phase of the cell cycle. More error-prone polymerases also exist and have specific functions including DNA repair and cell recombination [43]. While it is imperative that the cells employ methods to preserve the fidelity of DNA replication, error-prone polymerases may play a role in producing variability which could provide a selective advantage [43]. As such, in 1962 Magni and 
von Borstel observed that cells had a markedly higher rate of mutation during meiosis compared to mitosis [44].

DNA polymerase $\beta$ (POL $\beta$ ) is one of the more error prone polymerases found in eukaryotes and is required for base excision repair during DNA replication and repair [45]. It has an error rate of one error per 10,000-100,000 nucleotides incorporated, up to $100 \mathrm{x}$ higher than the more accurate polymerases which the cell routinely uses for chromosomal replication [43]. Recently, POL $\beta$ has also been found to have a critical role during meiotic synapsis [45]. POL $\beta$ has been shown to localize to the synaptonemal complex during prophase I [46] where it facilitates the removal of the SPO11 complex from DNA breakage ends, facilitating the creation of ssDNA with which RAD51 and DMC1 associate [47]. Kidane et al. showed that POL $\beta$ knockdown spermatocytes were unable to undergo chromosome synapsis and, instead, underwent apoptosis during prophase I [47].

Deregulated expression of POL $\beta$ has been implicated in genomic instability in cancer [45]. Decreased POL $\beta$ expression has been detected in one fifth of tumors [48] and this decrease has also been demonstrated to promote tumorigenesis [49] presumably due to decreased DNA repair. Conversely, increased levels of POL $\beta$ have been noted in approximately one-third of tumors studied, with one sample showing a 286-fold increase [48]. Luo et al. used mouse embryonic fibroblasts to study the effect of varying levels of POL $\beta$ on genomic instability by looking at three endpoints: DNA strand breaks, chromosomal breakage, and gene mutation [45]. POL $\beta$ null cells showed a marked increase in genomic instability [45] due to their inability to repair DNA. POL $\beta$ overexpressing cells showed a high frequency of mutations, but only after the introduction of DNA damaging agents [45], most likely because POL $\beta$ was used to repair the DNA damage instead of one of its higher fidelity counterparts.

Servant et al. specifically studied the association between DNA POL $\beta$ and melanoma and found that POL $\beta$ was markedly overexpressed in melanoma tissue when compared to normal skin [50]. Mammalian cells which overexpressed POL $\beta$ had a 1.5-2-fold higher resistance to UV radiation, though the surviving cells had a 2.6-50-fold increase in mutations [50]. POL $\beta$ was able to repair both cyclobutane pyrimidine dimers (CPD) and pyrimidine-pyrimidone (6-4) photoproducts and had the ability to elongate the $3^{\prime}$ strand after dATP addition [50]. This is significant because this period of elongation serves as a potential source of DNA point mutations due to POL $\beta$ 's markedly lower rate of fidelity than other polymerases.

Although POL $\beta$ seems to be expressed at some level ubiquitously in all cells, it clearly plays an integral role in the meiotic pathways. It would be anticipated that cells activating meiosis pathways may exhibit increased POL $\beta$ activity and increased mutations.

\section{Cohesion: How Not to Let Go of Your Sister}

The decision is made to enter the meiotic cycle prior to replicating the DNA. During the replication of DNA for meiosis, the newly duplicated strands are held together by a ring of proteins called cohesins. The mitotic cohesin complex normally includes the structural maintenance (SMC) proteins SMC1 and SMC2, the kleisin protein SCC1/RAD21, and an accessory subunit, SCC3 [51, 52]. In meiosis RAD21 is replaced by REC8 [53].

REC8 serves a number of functions including (1) acting within the synaptonemal complex to drive homologous recombination, (2) kinetochore orientation, and (3) sister chromatid adhesion.

6.1. Synaptonemal Complex/Homologous Recombination. The synaptonemal complex is a unique meiosis specific structure which is critical for chiasma (locations of crossover) formation, homologous chromosome binding, and chromosome segregation [54]. It bridges homologous chromosomes and is composed of three proteins: SCP1, 2, and 3. REC8 is critical in this structure for driving chiasma formation between homologous chromosomes [55, 56]. As the chromosomes condense, REC8 is cleaved on the chromosome arms and the cohesin complex is replaced by the condensin complex. The crossover point remains to ensure proper alignment, tension, and segregation of the homologous chromosomes.

6.2. Kinetochore Orientation. REC8 has also been shown to play a role in the monoorientation of sister kinetochores during meiosis I. When the mitotic cohesin protein RAD21 is expressed instead of REC8 in yeast, sister chromatids undergo equational rather than reductional chromosomal segregation during meiosis I $[55,57]$. This suggests that the coorientation of kinetochores is lost, allowing sister chromatids to be pulled towards opposite poles of the dividing cell. Studies performed in maize support this theory, demonstrating that, in the absence of REC8, sister chromatids establish bioriented sister kinetochores in meiosis I [58]. While research suggests REC8 is required for monoorientation of sister kinetochores, its overexpression alone does not appear to always lead to chromosomal missegregation during mitosis $[55,57]$ but instead requires the cooperation of other factors such as monopolins, as reviewed below.

6.3. Kinetochore Adhesion. REC8 is retained around the centromere until the start of anaphase II [59] ensuring that sister chromatids do not become prematurely separated. Two of the factors that prevent the cleavage of REC8 at the centromere are SGO1 and SGO2. Although both of these proteins are ubiquitously expressed in mammals, SGO2 is found in higher concentrations in the testis, implying that it may play a more major role in meiosis [60]. Studies have supported this theory, showing that mice with a deleted Sgo2 gene show no failure to thrive but are infertile, suggesting that this protein is not as critical in mitosis as meiosis [61].

The protease separase is required to remove cohesins from chromosomes [62]. REC8 can only be cleaved by separase when the former is hyperphosphorylated [60]. SGO2 protects REC8 from cleavage during meiosis I by ensuring its dephosphorylation at the centromeres. This is accomplished through the recruitment of protein phosphatase 2A (PP2A) to centromere proximal cohesins [28]. PP2A localization to centromeres results in dephosphorylation of centromere proximal REC8, inhibiting its cleavage by separase while allowing REC8 cleavage to occur along chromosome arms. 
REC8/Synaptonemal complex proteins could play an important role in chromosomal missegregation and translocations. REC8 expression has been noted in melanoma [2] and we found it to be overexpressed in melanoma using western blot analysis (Figure 1) and immunofluorescence (Figure 2). REC8 has also been noted in irradiated lymphoma cells [22]. Forced expression of REC8 during mitosis leads to chromosome segregation defects [63]. Ishiguro et al. utilized yeast with overproduction of REC8 during mitosis to demonstrate this phenomenon [63]. Compared with the wild type yeast cells, the strains with upregulated REC8 showed bridged nuclei which were not able to divide appropriately [63]. Thus it is possible that high levels of REC8 may play a role in nuclear division abnormalities.

It has also been postulated that REC8 may play a role in reducing chromosome number in polyploid cancer cells by driving reductional divisions [22]. Interestingly, synaptonemal complex proteins SCP1 and SCP3 have been shown to be upregulated in cancer and are associated with tumor progression and survival $[16,22,64]$. SCP1 is a known cancer/testis antigen [64]. Türeci et al. found that in melanoma, four of the 28 samples tested showed positive synaptonemal complex protein expression [16]. Studies have demonstrated that when inserted into nongerm cells, these proteins are still able to produce a synaptonemal complex [54]. Thus it is possible that these proteins play a role in chromosomal missegregation and crossover events in cancer.

\section{Kinetochore/Spindle Assembly: A Different Orientation}

The kinetochore is a proteinaceous structure which forms on centromeres and serves as a spindle fiber attachment site used to pull chromosomes apart and to opposite poles of the nucleus during cell division. Every chromatid has its own kinetochore, thus after the duplication of genetic material each chromosome contains two kinetochores, one on each chromatid. During mitosis, the two kinetochores on each chromosome face in opposite directions so that spindle fibers from each pole attach to separate chromatids. This is known as amphitelic attachment. The kinetochore works as a sensing mechanism, ensuring that the chromosomes are appropriately amphitelically attached. It does this by detecting the tension produced from the counteracting pull from each spindle into the bidirectionally oriented sister kinetochores. Only when this tension is sensed does the cell proceed through the spindle assembly checkpoint (SAC) and begin anaphase.

This process is more complicated in meiosis, as the cell must undergo two subsequent rounds of chromosome segregation without intervening DNA duplication. This is accomplished by a different orientation of the kinetochores in meiosis I through the use of REC8, monopolin proteins, and Aurora B Kinase [58, 65]. In meiosis I, sister kinetochores are oriented on the same side of the chromosome (monooriented) and attach to spindle fibers in a syntelic fashion to ensure that sister chromatids are pulled towards the same pole of the dividing cell. The tension necessary to proceed through the checkpoint is created by the crossovers between homologous chromosomes, which are pulled in opposite directions. Resolution of the chiasma allows for separation of the joined homologous pairs in anaphase I. Meiosis II then proceeds similarly to mitosis.

Many of the details on kinetochore orientation and rotation in meiosis II have yet to be determined. REC8 is critical for mono-orientation $[55,58]$. Condensins, proteins which play a major role in chromosome condensation and DSB repair during prophase I, are also known to play a role [66]. Brito et al. showed that in the absence of condensins, a portion of kinetochores biorient during meiosis I [66]. A third group of proteins called monopolins also play a key role [6769]. Monopolins are meiosis specific proteins [69]. Through their interaction with Aurora B Kinase, monopolins help ensure that homologs are pulled towards opposite poles of the cell [67]. Although REC8 plays a clear role in maintaining monooriented sister kinetochores, monopolins alone are able to hold together sister kinetochores independently $[67,69]$.

Another critical part of this process is the spindle apparatus. In the female oocyte, the centrosome is destroyed before meiosis and the cell undergoes an acentrosomal spindle assembly [70, 71]. The spindle network is instead formed through the action of over 80 self-organized microtubule organized centers (MTOCs) that develop from the cytoplasmic microtubule complex and eventually aggregate into a bipolar network [70]. Very little is understood about acentrosomal spindle assembly and the differences between the mitotic and meiotic spindle, and further studies are needed.

During meiosis I, mechanisms exist to allow the unique process of homolog separation. Studies have shown that some SAC proteins specifically interact with REC8 and Shugoshin (SGO1 and 2) [56]. BUB1 is one of the SAC proteins shown to be specifically involved with this process. BUB1 is required for the localization of the meiosis cohesion regulators SGO1 and SGO2 to protect REC8 during meiosis I [72]. For this reason, BUB1 is thought to be essential for establishing proper kinetochore function [56, 73, 74]. BUB1 mutation results in chromosome fragmentation and missegregation in Drosophila $[56,73]$ and female specific germ cell aneuploidy in mice $[56,75]$.

BUB1 has been noted to be abnormally expressed in several cancers including gastric, colon, esophageal, breast, and melanoma [76-79]. The aberrant expression of BUB1 seems to have an especially strong correlation with melanoma [80]. Lewis et al. used qtPCR to identify molecular expression patterns in melanoma, benign nevi, and lymph nodes [80]. Of the 20 melanoma-related genes tested, BUB1 was one of the three genes found to have the highest discriminatory potential for distinguishing melanoma, benign nevi, and lymph nodes [80].

There is a delicate interplay between kinetochores, the spindle apparatus, and the SAC which, when not functioning properly, would be expected to result in chromosomal segregation abnormalities.

\section{Conclusion and Future Directions}

Melanomas are highly genomically unstable tumors and are known to express germ cell proteins. It is possible that these two phenomena are related due to the collision of 
TABle 3: The Potential ramifications of the aberrant expression of different meiotic proteins.

\begin{tabular}{lcr}
\hline Meiotic Pathway & Potential ramification in mitosis & Potential Meiotic Proteins involved \\
\hline DS DNA strand breaks & Insertions, deletions, translocations & SPO11 \\
Error prone polymerase & Point mutations & POLB \\
Failure of cohesin ring digestion & Tetraplody, polyploidy & REC8/SGO2 \\
Unresolved chismata & Anaphase bridging & REC8 \\
Failure of kinetochore separation & Chromosomal missegregation & REC8/SGO2 \\
Misalignment of kinetochores & Chromosomal missegregation & REC8/monopolins \\
\hline
\end{tabular}

meiotic germ cell pathways with the normal mitotic cell cycle pathways (meiomitosis). As discussed, these pathways could impact genomic instability at many levels including double stranded DNA breaks, crossover events, chromosomal cohesion, spindle defects, and direct introduction of point mutations (Table 3). Chromosomal instability is a hallmark of human cancer, and we hypothesize that the aberrant expression of meiosis proteins will prove to be a critical step in this process. Expression of these pathways may serve a diagnostic role in identifying tumor with particular aggressive behavior due to their capacity to continuously evolve. Further, these pathways may serve as therapeutic targets. Because meiosis proteins are largely limited to germ cells, targeted therapeutics could be designed to have very limited interactions with other cells in the body reducing potential adverse side effects. Meiomitosis is likely to play a critical role in cancer progression and we believe future research is needed to shed light on this promising field.

\section{Disclosure}

Scott F. Lindsey, Diana M. Byrnes, Ashley M. Rosa, Nitika Dabas, Mark S. Eller, and James M. Grichnik do not have any financial relationships relevant to this paper. Grichnik is a DigitalDerm, Inc., Major Shareholder. Scott F. Lindsey, Diana M. Byrnes, Ashley M. Rosa, Nitika Dabas, Mark S. Eller do not have any other financial relationships.

\section{Funding}

This study was supported by the Anna Fund Melanoma Program at Sylvester Comprehensive Cancer Center, the Melanoma Research Foundation, and the Frankel Family Division of Melanocytic Tumors, Department of Dermatology and Cutaneous Surgery, University of Miami.

\section{Acknowledgments}

The authors are indebted to the Anna Fund Melanoma Program at Sylvester Comprehensive Cancer Center, the Frankel Family Division of Melanocytic Tumors, the Department of Dermatology and Cutaneous Surgery, the Melanoma Research Foundation, and our many benefactors, especially William Rubin and his family and friends.

\section{References}

[1] B. C. Bastian, A. B. Olshen, P. E. LeBoit, and D. Pinkel, "Classifying melanocytic tumors based on DNA copy number changes,"
American Journal of Pathology, vol. 163, no. 5, pp. 1765-1770, 2003.

[2] A. M. Rosa, N. Dabas, D. M. Byrnes, M. S. Eller, and J. M. Grichnik, "Germ cell proteins in melanoma: prognosis, diagnosis, treatment, and theories on expression," Journal of Skin Cancer, vol. 2012, Article ID 621968, 8 pages, 2012.

[3] T. Davoli and T. de Lange, "The causes and consequences of polyploidy in normal development and cancer," Annual Review of Cell and Developmental Biology, vol. 27, pp. 585-610, 2011.

[4] S. Negrini, V. G. Gorgoulis, and T. D. Halazonetis, "Genomic instability-an evolving hallmark of cancer," Nature Reviews Molecular Cell Biology, vol. 11, no. 3, pp. 220-228, 2010.

[5] D. Hanahan and R. A. Weinberg, "Hallmarks of cancer: the next generation," Cell, vol. 144, no. 5, pp. 646-674, 2011.

[6] D. Hanahan and R. A. Weinberg, "The hallmarks of cancer," Cell, vol. 100, no. 1, pp. 57-70, 2000.

[7] M. Roh, O. E. Franco, S. W. Hayward, R. van der Meer, and S. A. Abdulkadir, "A role for polyploidy in the tumorigenicity of Pim-1-expressing human prostate and mammary epithelial cells," PLoS ONE, vol. 3, no. 7, Article ID e2572, 2008.

[8] G. Brkic, J. Gopas, N. Tanic et al., "Genomic instability in drug-resistant human melanoma cell lines detected by Alu-Iarbitrary-primed PCR," Anticancer Research, vol. 23, no. 3, pp. 2601-2608, 2003.

[9] V. M. Draviam, S. Xie, and P. K. Sorger, "Chromosome segregation and genomic stability," Current Opinion in Genetics and Development, vol. 14, no. 2, pp. 120-125, 2004.

[10] D. Gilley, H. Tanaka, and B. S. Herbert, "Telomere dysfunction in aging and cancer," International Journal of Biochemistry and Cell Biology, vol. 37, no. 5, pp. 1000-1013, 2005.

[11] W. T. Silkworth, I. K. Nardi, L. M. Scholl, and D. Cimini, "Multipolar spindle pole coalescence is a major source of kinetochore mis-attachment and chromosome mis-segregation in cancer cells," PLoS ONE, vol. 4, no. 8, Article ID e6564, 2009.

[12] A. G. Silva, H. A. Graves, A. Guffei et al., "Telomere-centromere-driven genomic instability contributes to karyotype evolution in a mouse model of melanoma," Neoplasia, vol. 12, no. 1, pp. 11-19, 2010.

[13] E. Fratta, S. Coral, A. Covre et al., "The biology of cancer testis antigens: putative function, regulation and therapeutic potential," Molecular Oncology, vol. 5, no. 2, pp. 164-182, 2011.

[14] P. Chomez, O. de Backer, M. Bertrand, E. de Plaen, T. Boon, and S. Lucas, "An overview of the MAGE gene family with the identification of all human members of the family," Cancer Research, vol. 61, no. 14, pp. 5544-5551, 2001.

[15] S. Svobodová, J. Browning, D. MacGregor et al., "Cancertestis antigen expression in primary cutaneous melanoma has independent prognostic value comparable to that of Breslow thickness, ulceration and mitotic rate," European Journal of Cancer, vol. 47, no. 3, pp. 460-469, 2011. 
[16] Ö. Türeci, U. Sahin, C. Zwick, M. Koslowski, G. Seitz, and M. Pfreundschuh, "Identification of a meiosis-specific protein as a member of the class of cancer/testis antigens," Proceedings of the National Academy of Sciences of the United States of America, vol. 95, no. 9, pp. 5211-5216, 1998.

[17] Y. T. Chen, C. A. Venditti, G. Theiler et al., "Identification of CT46/HORMAD1, an immunogenic cancer/testis antigen encoding a putative meiosis-related protein," Cancer Immunity, vol. 5, p. 9, 2005.

[18] A. J. G. Simpson, O. L. Caballero, A. Jungbluth, Y. T. Chen, and L. J. Old, "Cancer/testis antigens, gametogenesis and cancer," Nature Reviews Cancer, vol. 5, no. 8, pp. 615-625, 2005.

[19] J. M. Grichnik, “Genomic instability and tumor stem cells," Journal of Investigative Dermatology, vol. 126, no. 6, pp. 1214-1216, 2006.

[20] J. M. Grichnik, "Melanoma, nevogenesis, and stem cell biology," Journal of Investigative Dermatology, vol. 128, no. 10, pp. 23652380, 2008.

[21] M. Kalejs and J. Erenpreisa, "Cancer/testis antigens and gametogenesis: a review and "brain-storming" session," Cancer Cell International, vol. 5, no. 1, article 4, 2005.

[22] M. Kalejs, A. Ivanov, G. Plakhins et al., "Upregulation of meiosis-specific genes in lymphoma cell lines following genotoxic insult and induction of mitotic catastrophe," BMC Cancer, vol. 6, article 6, 2006.

[23] E. Marcon and P. B. Moens, "The evolution of meiosis: recruitment and modification of somatic DNA-repair proteins," BioEssays, vol. 27, no. 8, pp. 795-808, 2005.

[24] H. Bernstein, F. A. Hopf, and R. E. Michod, "The molecular basis of the evolution of sex," Advances in Genetics, vol. 24, pp. 323370, 1987.

[25] H. Bernstein, H. C. Byerly, F. A. Hopf, and R. E. Michod, "Genetic damage, mutation, and the evolution of sex," Science, vol. 229, no. 4719, pp. 1277-1281, 1985.

[26] L. Hadany and J. M. Comeron, "Why are sex and recombination so common?" Annals of the New York Academy of Sciences, vol. 1133, pp. 26-43, 2008.

[27] S. P. Otto and A. C. Gerstein, "Why have sex? The population genetics of sex and recombination," Biochemical Society Transactions, vol. 34, no. part 4, pp. 519-522, 2006.

[28] G. A. Brar and A. Amon, "Emerging roles for centromeres in meiosis I chromosome segregation," Nature Reviews Genetics, vol. 9, no. 12, pp. 899-910, 2008.

[29] J. Kimble, "Molecular regulation of the mitosis/meiosis decision in multicellular organisms," Cold Spring Harbor Perspectives in Biology, vol. 3, no. 8, Article ID a002683, 2011.

[30] Y. Watanabe, S. Shinozaki-Yabana, Y. Chikashige, Y. Hiraoka, and M. Yamamoto, "Phosphorylation of RNA-binding protein controls cell cycle switch from mitotic to meiotic in fission yeast," Nature, vol. 386, no. 6621, pp. 187-190, 1997.

[31] J. Kimble and S. L. Crittenden, "Controls of germline stem cells, entry into meiosis, and the sperm/oocyte decision in Caenorhabditis elegans," Annual Review of Cell and Developmental Biology, vol. 23, pp. 405-433, 2007.

[32] C. R. Eckmann, S. L. Crittenden, N. Suh, and J. Kimble, "GLD3 and control of the mitosis/meiosis decision in the germline of Caenorhabditis elegans," Genetics, vol. 168, no. 1, pp. 147-160, 2004.

[33] E. L. Anderson, A. E. Baltus, H. L. Roepers-Gajadien et al., "Stra8 and its inducer, retinoic acid, regulate meiotic initiation in both spermatogenesis and oogenesis in mice," Proceedings of the National Academy of Sciences of the United States of America, vol. 105, no. 39, pp. 14976-14980, 2008.

[34] H. Li, K. Palczewski, W. Baehr, and M. Clagett-Dame, "Vitamin A deficiency results in meiotic failure and accumulation of undifferentiated spermatogonia in prepubertal mouse testis," Biology of Reproduction, vol. 84, no. 2, pp. 336-341, 2011.

[35] Y. Watanabe, S. Yokobayashi, M. Yamamoto, and P. Nurse, "Pre-meiotic $S$ phase is linked to reductional chromosome segregation and recombination," Nature, vol. 409, no. 6818, pp. 359-363, 2001.

[36] J. L. Gerton and R. S. Hawley, "Homologous chromosome interactions in meiosis: diversity amidst conservation," Nature Reviews Genetics, vol. 6, no. 6, pp. 477-487, 2005.

[37] S. Keeney, "Spoll and the formation of DNA double-strand breaks in meiosis," Genome Dynamics and Stability, vol. 2, pp. 81-123, 2008.

[38] V. Borde, "The multiple roles of the Mrell complex for meiotic recombination," Chromosome Research, vol. 15, no. 5, pp. 551563, 2007.

[39] H. Niu, L. Wan, B. Baumgartner, D. Schaefer, J. Loidl, and N. M. Hollingsworth, "Partner choice during meiosis is regulated by Hopl-promoted dimerization of Mek1," Molecular Biology of the Cell, vol. 16, no. 12, pp. 5804-5818, 2005.

[40] H. Niu, L. Wan, V. Busygina et al., "Regulation of meiotic recombination via Mek1-mediated Rad54 phosphorylation," Molecular Cell, vol. 36, no. 3, pp. 393-404, 2009.

[41] H. Niu, X. Li, E. Job et al., "Mek1 kinase is regulated to suppress double-strand break repair between sister chromatids during budding yeast meiosis," Molecular and Cellular Biology, vol. 27, no. 15, pp. 5456-5467, 2007.

[42] K. Daniel, J. Lange, K. Hached et al., "Meiotic homologue alignment and its quality surveillance are controlled by mouse HORMAD1," Nature Cell Biology, vol. 13, no. 5, pp. 599-610, 2011.

[43] A. J. Rattray and J. N. Strathern, "Error-prone DNA polymerases: when making a mistake is the only way to get ahead," Annual Review of Genetics, vol. 37, pp. 31-66, 2003.

[44] G. E. Magni and R. C. von Borstel, "Different rates of spontaneous mutation during mitosis and meiosis in yeast," Genetics, vol. 47, no. 8, pp. 1097-1108, 1962.

[45] Q. Luo, Y. Lai, S. Liu, M. Wu, Y. Liu, and Z. Zhang, "Deregulated expression of DNA polymerase beta is involved in the progression of genomic instability," Environmental and Molecular Mutagenesis, vol. 53, no. 5, pp. 325-333, 2012.

[46] A. W. Plug, C. A. Clairmont, E. Sapi, T. Ashley, and J. B. Sweasy, "Evidence for a role for DNA polymerase $\beta$ in mammalian meiosis," Proceedings of the National Academy of Sciences of the United States of America, vol. 94, no. 4, pp. 1327-1331, 1997.

[47] D. Kidane, A. S. Jonason, T. S. Gorton et al., "DNA polymerase beta is critical for mouse meiotic synapsis," The EMBO Journal, vol. 29, no. 2, pp. 410-423, 2010.

[48] N. Bhattacharyya, H. C. Chen, L. Wang, and S. Banerjee, "Heterogeneity in expression of DNA polymerase $\beta$ and DNA repair activity in human tumor cell lines," Gene Expression, vol. 10, no. 3, pp. 115-123, 2002.

[49] V. Poltoratsky, R. Prasad, J. K. Horton, and S. H. Wilson, "Down-regulation of DNA polymerase $\beta$ accompanies somatic hypermutation in human BL2 cell lines," DNA Repair, vol. 6, no. 2, pp. 244-253, 2007.

[50] L. Servant, C. Cazaux, A. Bieth, S. Iwai, F. Hanaoka, and J.-S. Hoffmann, "A role for DNA polymerase $\beta$ in mutagenic UV 
lesion bypass," The Journal of Biological Chemistry, vol. 277, no. 51, pp. 50046-50053, 2002.

[51] K. I. Ishiguro and Y. Watanabe, "Chromosome cohesion in mitosis and meiosis," Journal of Cell Science, vol. 120, no. part 3, pp. 367-369, 2007.

[52] K. Nasmyth and C. H. Haering, "The structure and function of SMC and kleisin complexes," Annual Review of Biochemistry, vol. 74, pp. 595-648, 2005.

[53] S. L. Page and R. S. Hawley, "Chromosome choreography: the meiotic ballet," Science, vol. 301, no. 5634, pp. 785-789, 2003.

[54] R. Öllinger, M. Alsheimer, and R. Benavente, "Mammalian protein SCP1 forms synaptonemal complex-like structures in the absence of meiotic chromosomes," Molecular Biology of the Cell, vol. 16, no. 1, pp. 212-217, 2005.

[55] Y. Watanabe and P. Nurse, "Cohesin Rec8 is required for reductional chromosome segregation at meiosis," Nature, vol. 400, no. 6743, pp. 461-464, 1999.

[56] S. C. Sun and N. H. Kim, "Spindle assembly checkpoint and its regulators in meiosis," Human Reproduction Update, vol. 18, no. 1, pp. 60-72, 2012.

[57] S. Yokobayashi, M. Yamamoto, and Y. Watanabe, "Cohesins determine the attachment manner of kinetochores to spindle microtubules at meiosis I in fission yeast," Molecular and Cellular Biology, vol. 23, no. 11, pp. 3965-3973, 2003.

[58] S. Hauf and Y. Watanabe, "Kinetochore orientation in mitosis and meiosis," Cell, vol. 119, no. 3, pp. 317-327, 2004.

[59] M. Eijpe, H. Offenberg, R. Jessberger, E. Revenkova, and C. Heyting, "Meiotic cohesin REC8 marks the axial elements of rat synaptonemal complexes before cohesins SMC1 $\beta$ and SMC3," Journal of Cell Biology, vol. 160, no. 5, pp. 657-670, 2003.

[60] Y. Yao and W. Dai, "Shugoshins function as a guardian for chromosomal stability in nuclear division," Cell Cycle, vol. 11, no. 14, pp. 2631-2642, 2012.

[61] E. Llano, R. Gómez, C. Gutiéerrez-Caballero et al., "Shugoshin2 is essential for the completion of meiosis but not for mitotic cell division in mice," Genes and Development, vol. 22, no. 17, pp. 2400-2413, 2008.

[62] N. R. Kudo, M. Anger, A. H. F. M. Peters et al., "Role of cleavage by separase of the Rec8 kleisin subunit of cohesin during mammalian meiosis I," Journal of Cell Science, vol. 122, no. part 15, pp. 2686-2698, 2009.

[63] T. Ishiguro, K. Tanaka, T. Sakuno, and Y. Watanabe, "ShugoshinPP2A counteracts casein-kinase-1-dependent cleavage of Rec8 by separase," Nature Cell Biology, vol. 12, no. 5, pp. 500-506, 2010 .

[64] J. Tammela, A. A. Jungbluth, F. Qian et al., "SCP-1 cancer/testis antigen is a prognostic indicator and a candidate target for immunotherapy in epithelial ovarian cancer," Cancer Immunity, vol. 4, p. 10, 2004.

[65] M. Petronczki, M. F. Siomos, and K. Nasmyth, "Un ménage à quatre: the molecular biology of chromosome segregation in meiosis," Cell, vol. 112, no. 4, pp. 423-440, 2003.

[66] I. L. Brito, H. G. Yu, and A. Amon, "Condensins promote coorientation of sister chromatids during meiosis I in budding yeast," Genetics, vol. 185, no. 1, pp. 55-64, 2010.

[67] F. Monje-Casas, V. R. Prabhu, B. H. Lee, M. Boselli, and A. Amon, "Kinetochore orientation during meiosis is controlled by Aurora B and the monopolin complex," Cell, vol. 128, no. 3, pp. 477-490, 2007.

[68] I. L. Brito, F. Monje-Casas, and A. Amon, "The Lrs4-Csm1 monopolin complex associates with kinetochores during anaphase and is required for accurate chromosome segregation," Cell Cycle, vol. 9, no. 17, pp. 3611-3618, 2010.

[69] A. Tóth, K. P. Rabitsch, M. Gálová, A. Schleiffer, S. B. C. Buonomo, and K. Nasmyth, "Functional genomics identifies monopolin: a kinetochore protein required for segregation of homologs during meiosis I," Cell, vol. 103, no. 7, pp. 1155-1168, 2000.

[70] M. Schuh and J. Ellenberg, "Self-organization of MTOCs replaces centrosome function during acentrosomal spindle assembly in live mouse oocytes," Cell, vol. 130, no. 3, pp. 484498, 2007.

[71] J. Dumont and A. Desai, "Acentrosomal spindle assembly and chromosome segregation during oocyte meiosis," Trends in Cell Biology, vol. 22, no. 5, pp. 241-249, 2012.

[72] T. S. Kitajima, S. A. Kawashima, and Y. Watanabe, "The conserved kinetochore protein shugoshin protects centromeric cohesion during meiosis," Nature, vol. 427, no. 6974, pp. 510-517, 2004.

[73] J. Basu, H. Bousbaa, E. Logarinho et al., "Mutations in the essential spindle checkpoint gene Bubl cause chromosome missegregation and fail to block apoptosis in Drosophila," Journal of Cell Biology, vol. 146, no. 1, pp. 13-28, 1999.

[74] S. Yamaguchi, A. Decottignies, and P. Nurse, "Function of Cdc2p-dependent Bublp phosphorylation and Bublp kinase activity in the mitotic and meiotic spindle checkpoint," The EMBO Journal, vol. 22, no. 5, pp. 1075-1087, 2003.

[75] S. Leland, P. Nagarajan, A. Polyzos et al., "Heterozygosity for a Bub1 mutation causes female-specific germ cell aneuploidy in mice," Proceedings of the National Academy of Sciences of the United States of America, vol. 106, no. 31, pp. 12776-12781, 2009.

[76] C. Klebig, D. Korinth, and P. Meraldi, "Bub1 regulates chromosome segregation in a kinetochore-independent manner," Journal of Cell Biology, vol. 185, no. 5, pp. 841-858, 2009.

[77] H. Shigeishi, N. Oue, H. Kuniyasu et al., "Expression of Bubl gene correlates with tumor proliferating activity in human gastric carcinomas," Pathobiology, vol. 69, no. 1, pp. 24-29, 2001.

[78] M. Shichiri, K. Yoshinaga, H. Hisatomi, K. Sugihara, and Y. Hirata, "Genetic and epigenetic inactivation of mitotic checkpoint genes hBUB1 and hBUBR1 and their relationship to survival," Cancer Research, vol. 62, no. 1, pp. 13-17, 2002.

[79] S. H. Doak, G. J. S. Jenkins, E. M. Parry, A. P. Griffiths, J. N. Baxter, and J. M. Parry, "Differential expression of the MAD2, BUB1 and HSP27 genes in Barrett's oesophagus-their association with aneuploidy and neoplastic progression," Mutation Research, vol. 547, no. 1-2, pp. 133-144, 2004.

[80] T. B. Lewis, J. E. Robison, R. Bastien et al., "Molecular classification of melanoma using real-time quantitative reverse transcriptase-polymerase chain reaction," Cancer, vol. 104, no. 8, pp. 1678-1686, 2005. 


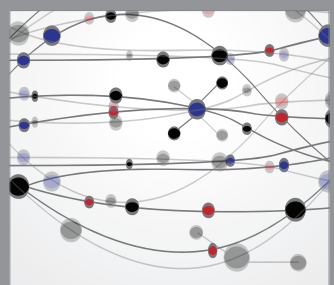

The Scientific World Journal
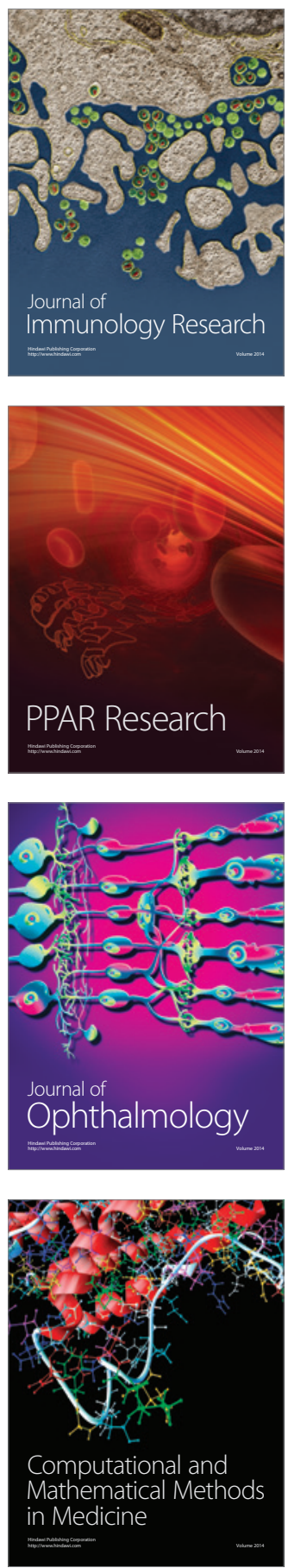

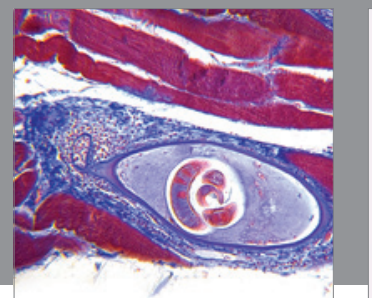

Gastroenterology

Research and Practice
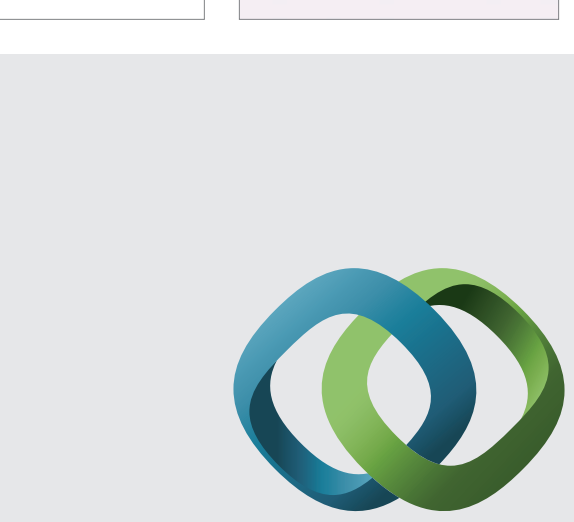

\section{Hindawi}

Submit your manuscripts at

http://www.hindawi.com
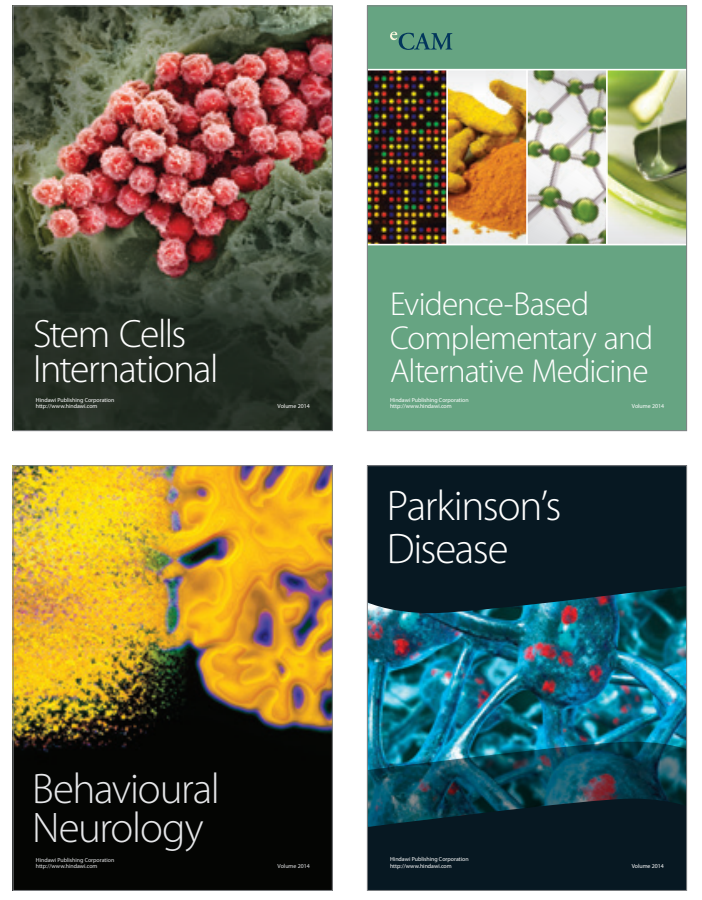
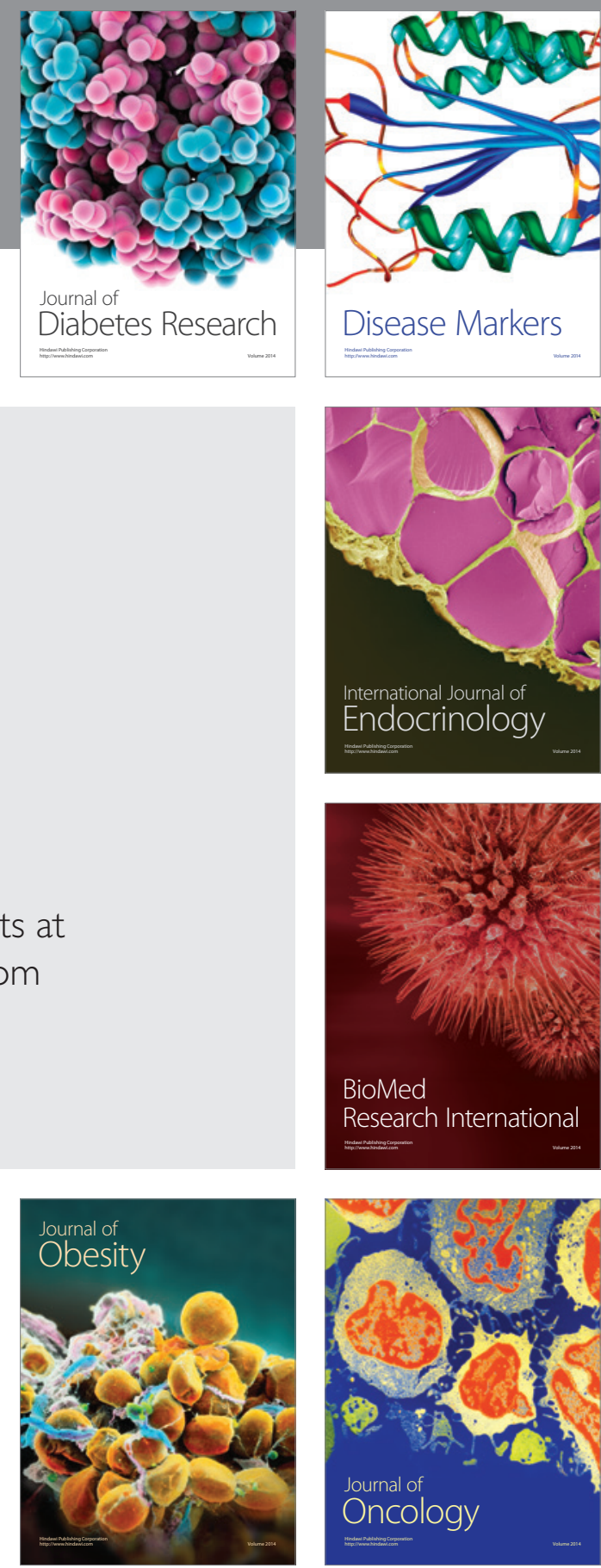

Disease Markers
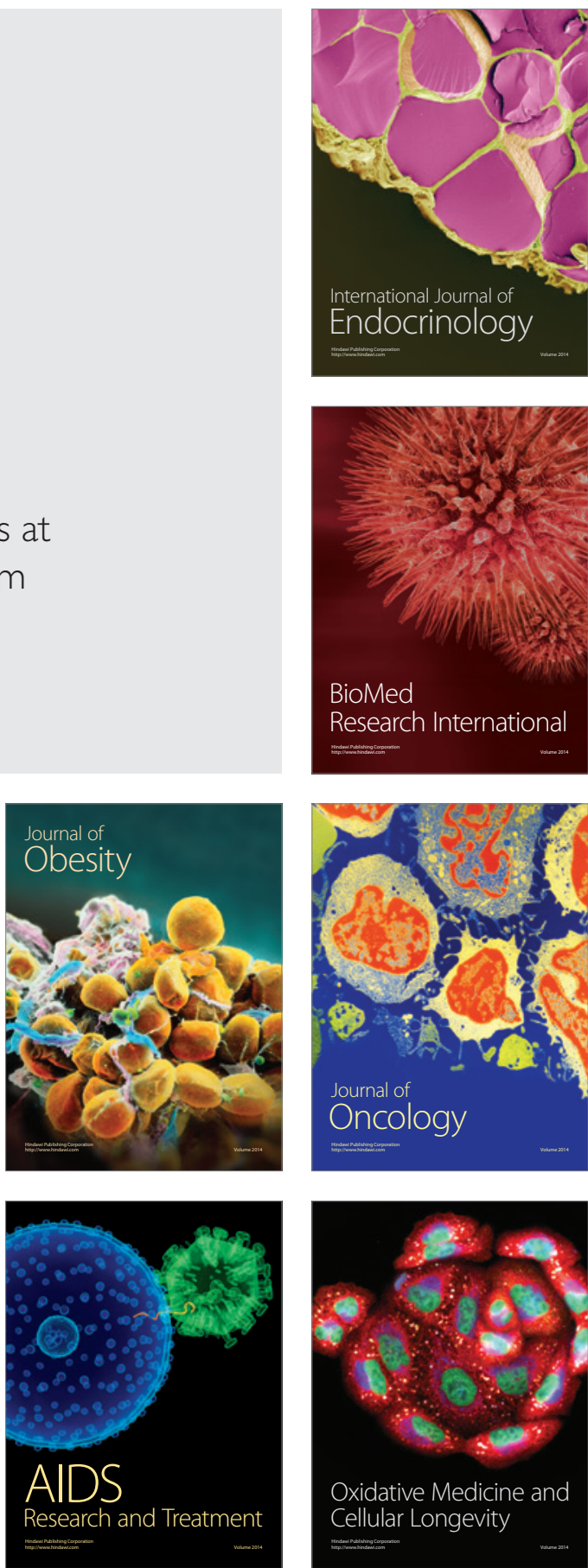